\section{Maria Piane \\ Patrizia Lulli \\ Ivano Farinelli \\ Simona Simeoni \\ Sergio De Filippis \\ Francesca Romana Patacchioli \\ Paolo Martelletti}

\title{
Genetics of migraine and pharmacogenomics: some considerations
}

Received: 1 September 2007

Accepted in revised form: 20 November 2007

Published online: 17 December 2007

${ }^{\circ} 2007$ The Author ${ }^{1}$

\section{Piane (凶) • P. Lulli}

Department of Diagnostic Sciences, Medical Genetics Section,

$2^{\text {nd }}$ School of Medicine,University

of Rome La Sapienza, I-00185, Rome, Italy

e-mail: maria.piane@uniroma1.it

Tel.: +39-06-3377-5262

Fax: +39-06-3377-5422

I. Farinelli • S. De Filippis • P. Martelletti Department of Medical Sciences,

$2^{\text {nd }}$ School of Medicine, University of Rome

La Sapienza, Internal Medicine,

Regional Referral Headache Centre,

Sant'Andrea Hospital, Rome, Italy

S. Simeoni • F. R. Patacchioli

Department of Human Physiology and

Pharmacology, $2^{\text {nd }}$ School of Medicine,

Sapienza University of Rome, Rome, Italy
Abstract Migraine is a complex disorder caused by a combination of genetic and environmental factors.

Although family and twin studies show that there is a genetic component in migraine, no genes predisposing to common forms of the disorder, migraine with and without aura, have been identified. Patients with migraine respond differently to a given drug administered. The efficacy of therapy and the occurrence of adverse drug response are a consequence of individual variability. Genetic profiling of predisposition to migraine should facilitate the development of more effective diagnostic and therapeutic applications. The development of International Hap Map project could provide a powerful tool for identification of the candidate genes in this complex disease and pharmacogenomics research could be the promise for individualized treatments and prevention of adverse drug response.

Keywords Pharmacogenomics • Pharmacogenetics • Non-responder patients $\cdot$ Migraine

\section{Introduction}

Migraine is a complex disorder caused by a combination of genetic and environmental factors. At present, the measure in which these two factors influence each other is unknown. It is possible to define this disease as a complex spectrum includ- ing features such as craniofacial pain, autonomic dysfunctions, mood disregulations and spreading cortical disturbance represented by both positive and negative aura phenomena [1-5].

Migraine diagnosis is based on anamnesis, a record patient's symptomatology and the exclusion of secondary causes. A positive familial history often represents further

'This is a "Springer Open Choice" article. Unrestricted non-commercial use, distribution, and reproduction in any medium is permitted, provided the original author and source are credited. 
evidence of this diagnosis. A more precise determination of familial aggregation appears in the calculation of disease relative risk of the population in specified groups of relatives. Epidemiological surveys found that first-degree relatives of probands displayed a much increased risk of developing the disorder of proband as compared to the general population [5]. The risk increase in close relatives obviously does not imply the pathology's manifestation but only the sharing of a genotypic pattern, which determines the outbreak of clinical manifestations after environmental stimuli of different kinds (stress factors, food, smells).

At present, the International Headache Society (IHS) classification recognises some phenotypical features attributing them to diagnostic subtypes. However, patients with the same diagnosis present a symptomatological pattern (type of pain, pain location, accompanying symptoms) underlining a great variability of expressions. According to this view, migraine could represent a syndrome whose clinical components are the reflection of specific loci expressions, independently influencing susceptibility to migraine [6].

The family aggregation of migraine with aura (MA) and migraine without aura (MO) evidence that there is a genetic component as the basis of migraine susceptibility. In a Finnish study involving 2690 monozygotic and 5497 dizygotic twin pairs, the genetic component ranged from $34 \%$ to $51 \%$ in different migraine types, without remarkable variations with respect to gender. Such a concordance emphasises the multifactorial nature of this pathology [7]. Similar results have been obtained in several studies on adult twin pairs [8-10].

Although family and twin studies show that there is a genetic component in migraine, no genes predisposing to common forms of the disorder have been identified. At present, several loci 4q21-q24, 5q21, 6p12.2-p21.1, 11q24, $14 \mathrm{q} 21.2-\mathrm{q} 22.3$ and 15q11-q13 linked to common forms of migraine with evidence of linkage have been reported. Several genes were described involved in migraine in many studies, but always with controversial results. In a more general scenario, genetic studies on primary headaches constitute challenges made more complex by this pathology.

Patients with migraine and headache respond differently to a given drug administered. The efficacy of a drug therapy and the occurrence of adverse reactions are a direct consequence of individual variability. Most of this variation in individual response is genetically determined and is the object of the study of pharmacogenetics.

\section{Familial hemiplegic migraine, a Mendelian model for migraine}

At present, the best genetic evidence providing molecular insight into migraine comes from the mutations that have been detected in a rare Mendelian form of migraine, named familial hemiplegic migraine (FHM) [11]. This represents the only gene-related headache disorder that has been identified so far.

FHM is a rare subtype of MA with an autosomal dominant inheritance pattern; it is a primary headache form characterised by motor weakness (transient hemiparesis or hemiplegia) during the aura phase of migraine attacks, and similar episodes in at least one first- or second-degree family member. Migraine symptoms are similar to those of "normal" migraine patients. FHM is genetically heterogeneous. Mutations in three different genes have been identified in FHM families: CACNA1A gene, involved in FHM type 1 (FHM1), localised to chromosome 19p13, encoding for the $\alpha 1 \mathrm{~A}$ subunit of the neuronal voltage-gated calcium channel $\mathrm{Cav}_{2} .1$ (neuronal P/Q-type calcium channel) [12]; ATP1A2 gene localised to chromosome 1q21-23, implicated in FHM type 2 (FHM2) encoding for the $\alpha 2$ subunit of the $\mathrm{Na}^{+}, \mathrm{K}^{+}-$ ATPase isoform [13]; and SCN1A gene localised to chromosome 2q24, implicated in FHM type 3 (FHM3), encoding a neuronal voltage-gated sodium channel $\left(\mathrm{Na}_{v} 1.1\right)$ [14]. All of them are expressed at cerebral level. Patients affected by FHM present a symptomatic range of which migraine constitutes just one aspect. In fact, some FHM families can present with only cerebellar degeneration or seizures and in the same way several genes, isolated in FHM families, are also involved in other neurological disorders with an autosomal dominant inheritance pattern such as episodic ataxia type 2 and spinocerebellar ataxia type $6[15,16]$.

Functional studies of FHM support the hypothesis that ion transport dysfunction is one of the main factors determining susceptibility of the brain to migraine attacks. New therapeutic strategies are aimed at controlling cortical hyperexcitability to prevent clinical manifestations: the protracted administration of some anti-migraine drugs would appear to change the expression of the genes.

Thus, the genes involved in FHM pathogenesis could be possible therapeutic targets, although the many studies investigating the role of CACNAIA gene in common forms of migraine have produced contradictory results $[17,18]$.

More generally, the role of FHM genes codifying for neuronal channels as $\mathrm{Cav}_{2} .1, \mathrm{Na}_{v} 1, \mathrm{Na}^{+}$and $\mathrm{K}^{+}$-ATPase at the cerebral level constitute a possible object of pharmacogenomic research [19].

This is true for FHM, but it seems to be far from a common form of migraine.

\section{Candidate genes and polymorphisms involved in migraine}

Although the three FHM genes represent the main candidate 
genes for understanding migraine pathogenesis also in the more common forms, several case-control association studies have indicated additional susceptibility genes implicated in migraine with and without aura [20-27]. Most of the genes identified to be associated to migraine need to be confirmed by further studies, because of the heterogeneity of the sample in terms of diagnosis, ethnic background and sample size, before being able to consider them as predisposing genes.

The C677T polymorphism of the methylenetetrahydrofolate reductase (MTHFR) gene has been suggested to be associated with MA in several populations [28], although recently in a large cohort of patients and controls this association failed to be verified. Nevertheless the same study does not confirm the positive association of the variants of ESR1 (oestrogen receptor $\alpha$ gene) with the migraine precedent described [20, 29].

McCarthy et al. [22] identified five single-nucleotide polymorphisms (SNPs) within the insulin receptor gene that showed a significant association with migraine, suggesting possible functions for the insulin receptor in the pathogenesis of this disease. The association between common migraine forms and the HLA system today represents one of the most investigated aspects.

In a group of Italian migraine patients, Martelletti et al. [23] showed that the frequency of HLA DR2 antigen decreased in the MA group when compared with both the MO and the control group. These results seemed to support the hypothesis of a protective role of DR2 antigen in MA and provided an additional basis for the proposed differences within $\mathrm{MO}$ and MA.

In a subsequent study conducted in a larger cohort of Italian patients, the associations of HLA DRB1 alleles and migraine were reported [24]. The frequency of the HLA DRB1*12 allele was found to be decreased in patients with migraine while the DRB1*16 allele, coding for HLA DR2 antigen, was significantly increased in comparison with controls. However once patients were divided into subgroups, MO patients presented a significant increase of the HLADRB*16 allele.

The discrepancy observed about DRB $1 * 12$ frequency in these studies can be due to the different sizes of the two samples, but both studies observed that the frequency of HLA DR2 was different between MA and MO groups, emphasising that there is a different genetic background in the two subtypes of migraine. A possible explanation for the association of HLA DRB1 alleles with migraine lies in the fact that this locus may be involved in susceptibility or may be in linkage disequilibrium with other genes, because the $D R B 1$ gene is located on chromosome 6p21, within the HLA class II region.

Recently, a significant association has been found between migraine and the polymorphisms of two genes: tumour necrosis factor- $\alpha$ (TNFA) and lymphotoxin $\alpha(T N F B)$, localised in the HLA class III region. TNF- $\alpha$ is a proinflammatory cytokine involved in both the outset and regulation of inflammatory response. The association of the TNFA 308G/A polymorphism with the occurrence of migraine has been shown only in MO patients; patients homozygous for the $\mathrm{G}$ allele present a higher risk of developing the disease. This suggests that the TNFA gene or a locus in linkage disequilibrium may be implicated in the risk of disorder modulation [25].

$T N F B$ codifies for a cytokine involved in the formation of secondary lymphoid organs during development and playing a role in apoptosis. The Nco1 polymorphism in the first intron of the $T N F B$ gene (TNFB*1 and TNFB*2 alleles) in migraine has been investigated. The frequency of the TFNB $* 2$ allele is significantly higher in MO patients, as compared with the control group, but no significant differences have been found between MA patients and controls. This evidence suggests a possible role of lymphotoxin $\alpha$ in the genetic background of migraine [26].

Given the possible role played by TNF genes and HLA, the task of the cytotoxic T lymphocyte antigen 4 (CTLA-4) has also been explored as they are negative regulators of $\mathrm{T}$ cell proliferation and therefore of cytokine production. Nevertheless, the results of the survey on CTLA-4 polymorphism $49 \mathrm{~A} / \mathrm{G}$ intron 1 failed to identify any positive association in MO and MA patients [27].

\section{Genetic bases of CDH and MOH}

Another aspect that has been investigated is the relationship between genetic factors and the possibility of developing chronic forms of headache (chronic daily headache, $\mathrm{CDH}$ ).

In MA patients, Peroutka et al. [30] explored the NcoI C/T polymorphism (His313His) in exon 6 of the dopamine receptor 2 gene (DRD2), located on chromosome 11q22. The patients with the DRD2 NcoI C/C genotype presented a higher incidence of MA episodes, major depression and anxiety disorders in comparison with individuals with a $\mathrm{T}$ allele. Paterna et al. [31] showed an increase in the frequency of migraine attacks in patients with angiotensin-converting enzyme (ACE) allele D polymorphisms localised in 17q23.

$\mathrm{CDH}$ patients are predisposed to developing drug abuse (medication overuse headache, $\mathrm{MOH}$ ) [32]. Several studies analysed this specific aspect in relation to genetic patterns. In these patients a role of the dopamine metabolism-related gene has been hypothesised. Polymorphisms of the dopamine transporter $(D A T)$ gene, which encodes a protein responsible for presynaptic uptake of dopamine, are involved in the development of drug abuse. Polymorphisms of the dopamine receptor $4(D R D 4)$ gene are implicated in the predisposition 
to episodic migraine attacks but not in drug abuse [33].

The Wolfram gene (WFS1) is located on chromosome 4 p16.1 and codifies for Wolframin, a transmembrane protein expressed in all cell types, in the brain predominantly in the limbic system or in structures closely related to it. Mutations in this gene are associated to Wolfram syndrome, a neurodegenerative disorder related to diabetes mellitus, diabetes insipidus, hearing loss, progressive blindness and a heterogeneous combination of psychiatric disorders. Thus, in $\mathrm{MOH}$ patients that show both an increase in abuse of symptomatic drugs and major psychiatric disorders, the His611Arg polymorphism of the WFS1 gene has been investigated. Individuals harbouring the R/R ( $\arg / \mathrm{arg}$ ) genotype showed significantly higher monthly drug consumption and more severe depressive symptoms than non-R/R individuals. This genetic substrate could constitute a possible marker of prognostic differentiation in $\mathrm{MOH}$ patients [34].

\section{Pharmacogenomics in migraine}

Patients with migraine and headache respond differently to given drugs. The efficacy of a drug therapy and the occurrence of adverse reactions are a direct consequence of individual variability, which is a complex trait depending on both genetic background (the genetic component present in each of us) and environmental factors [35, 36]. Most of this variation in individual response is genetically determined and is the object of the study of pharmacogenetics. Genetic factors influence the pharmacokinetics (absorption, distribution, metabolism and excretion of the drug) and pharmacodynamics (drug effect); the genetic variability determines the different ways in which a drug exerts its effect, for example by influencing the link with the target of the drug on which it acts; moreover it could make receptors, enzymes and proteins, involved in metabolism of a drug, different in structure and function.

Pharmacogenomics deals with the DNA and RNA variations related to a drug response; the gene expression study can be used as a diagnostic tool to predict whether a patient will respond to a drug well or badly, or not at all [37-39]. The realistic promises of migraine pharmacogenomic-based therapies are the advent of "individually-tailored drugs" and the potential to reduce costs not only in terms of money but also in human suffering and lost productivity [40]. Nowadays a great deal of scientific literature regarding genetic studies applied to migraine treatment can be found [41-44].

Pharmacogenetic information might be applied for identification of cases where certain drugs are simply not effective. In fact, pharmacogenomic factors can modify the drug response of an organism through a pharmacokinetic and phar- macodynamic action. The enzymes that metabolise drugs could present polymorphisms able to profoundly influence the dose-response relation among different patients. Knowledge of these polymorphisms is necessary for many drugs before starting a therapy.

Genetic profiling of predisposition to migraine should facilitate the development of more effective diagnostic and therapeutic applications.

Possible targets of pharmacogenomic research are both the serotoninergic and dopaminergic systems [38, 45]. The role of the serotoninergic system is suggested by the use of triptans in acute treatment. Triptans are drugs that act selectively as agonists on 5-HT1B/1D receptors producing vasoconstriction of meningial vessels as well as direct action on central pathways of trigeminal transmission [46, 47]. In several studies the relationship between polymorphisms of 5$\mathrm{HT} 1 \mathrm{~B}$ receptors and clinical response to sumatriptan has been investigated, however without being able to establish an unquestionable link between them [48]. The serotoninergic system's role is also suggested by the use of antidepressants, such as selective serotonin reuptake inhibitor (SSRI), in prophylaxis treatment [49].

Several studies indicate that hypersensitivity of the dopaminergic system is implicated in the pathogenesis of migraine [50]. In fact, as reported above, an association between $D R D 2$ gene and MA has been demonstrated [44, 51].

Moreover, some accompanying symptoms of migraine are mediated by the dopaminergic system (nausea, vomiting), underlining a possible role in clinical susceptibility to migraine.

\section{Adverse drug response and pharmacogenomics}

In terms of drug efficacy and toxicity, individual variability is associated with genetic variations in absorption and distribution into the site of action, or more generally in drug metabolism. In the same way the field can be extended to genes encoding drug transporters, drug receptors and other drug targets.

However, the majority of genetic factors involved in the variability of therapeutic effects and/or adverse drug response (ADR) are unknown and so far no molecular genetic clues have been identified for the more common types of primary headaches.

In recent years it has become clear that genetic factors could greatly modify drug responses as well as increase the risk of occurrence of ADR. In a review that analysed studies carried out between 1995 and 2000, the association between ADR of some drugs and the presence of at least one enzyme with a variant allele known to cause poor metabolism has 
been evidenced. These results suggest that a therapy based on genetic information could turn out to be clinically effective in the reduction of adverse outcomes [52].

If genetic information such as the allelic variants of enzymes involved in the metabolism was known, the choice of a therapy could be effectuated more precisely. However, the complex metabolic pathways of several drugs must be taken into account.

\section{Conclusions}

At the moment it is difficult to identify the best model for pharmacogenomics studies. Linkage studies failed to identify a valid model for the study of common forms of migraine.

Until now, only one or few SNPs have been analysed in most studies of genetic association in migraine. This approach has often led to conflicting results, as a consequence of using both different protocols and heterogeneous samples regarding ethnicity and size. The development of the International HapMap project [53] could also provide a powerful tool for identification of candidate genes in this complex disease [54]. Most chromosomes carry one of a few common ancestral haplotypes. These haplotypes in which SNPs are grouped in linkage disequilibrium blocks (tag SNP), identified by the HapMap project, can be used for the association study of genes as candidates for the presence of common variants that confer susceptibility to the disease. If a genetic variant on an ancestral chromosome increases risk of disease, the related ancestral haplotype will also be associated to the disease. So, tag SNP can be used as markers to identify the location of the disease variant, reducing genotyping costs and moreover standardising study design.

In the future, in addition to targeting a patient's drug concentrations within a therapeutic range, pharmacists are likely to be making dosage recommendations for individual drugs on the basis of the individual patient's genotype. As we enter the era of personalised drug therapy, we will be able to identify not only the best drug to be administered to a particular patient, but also the most effective and safest dosage from the outset of therapy.

Other advantages of genotyping over traditional therapeutic drug monitoring include the discovery of new targets for migraine therapy. Identification of polymorphisms and genetic biomarkers should help us to better understand migraine pathology and therefore choose the most effective treatments for migraine patients.

Acknowledgements This tutorial results from the lessons held at the Masters in Headache Medicine, Sapienza University of Rome, during the Academic Year 2006-2007 by the Authors (FRP, PL, PM). The Authors MP and PL contributed equally to this work.

\section{References}

1. Wessman M, Kaunisto MA, Kallela M, Palotie A (2004) The molecular genetics of migraine. Ann Med 36:462-473

2. Haan J, Kors EE, Vanmolkot KR et al (2005) Migraine genetics: an update. Curr Pain Headache Rep 9:213-220

3. Russell MB (2005) Tension-type headache in 40-year-olds: a Danish population-based sample of 4000. J Headache Pain 6:441-447

4. Russell MB (2006) Commentary to Comorbidity in Finnish migraine families. J Headache Pain 7:320-321

5. Russell MB (2007) Genetics in primary headaches. J Headache Pain 8:190-195

6. Anttila V, Kallela M, Oswell G et al (2006) Trait components provide tools to dissect the genetic susceptibility of migraine. Am J Hum Genet 79:85-99

7. Honkasalo ML, Kaprio J, Winter T et al (1995) Migraine and concomitant symptoms among 8167 adult twin pairs. Headache 35:70-78
8. Larsson B, Bille B, Pederson NL (1995) Genetic influence in headaches: a Swedish twin study. Headache 35:513-519

9. Gervil M, Ulrich V, Kyvik KO et al (1999) Migraine without aura: a population-based twin study. Ann Neurol 46:606-611

10. Ulrich V, Gervil M, Kyvik KO et al (1999) Evidence of a genetic factor in migraine with aura: a population-based Danish twin study. Ann Neurol 45:242-246

11. Colson NJ, Fernandez F, Lea RA, Griffiths LR (2007) The search for migraine genes: an overview of current knowledge. Cell Mol Life Sci 64:331-344

12. Ophoff RA, Terwindt GM, Vergouwe MN et al (1996) Familial hemiplegic migraine and episodic ataxia type- 2 are caused by mutations in the $\mathrm{Ca} 2+$ channel gene CACNL1A4. Cell 87:543-552
13. De Fusco M, Marconi R, Silvestri L et al (2003) Haploinsufficiency of ATP1A2 encoding the $\mathrm{Na}^{+} / \mathrm{K}^{+}$pump $\alpha 2$ subunit associated with familial hemiplegic migraine type 2. Nat Genet 33:192-196

14. Dichgans M, Freilinger T, Eckstein G et al (2005) Mutation in the neuronal voltage-gated sodium channel SCN1A in familial hemiplegic migraine. Lancet 366:371-377

15. Zhuchenko O, Bailey J, Bonnen P et al (1997) Autosomal dominant cerebellar ataxia (SCA6) associated with small polyglutamine expansions in the alpha 1A-voltage-dependent calcium channel. Nat Genet 15:62-69

16. Kors EE, Vanmolkot KRJ, Haan J et al (2004) Recent findings in headache genetics. Curr Opin Neurol 17:283-288 
17. Terwindt GM, Ophoff RA, van Eijk R et al Dutch Migraine Genetics Research Group (2001) Involvement of the CACNA1A gene containing region on 19 p13 in migraine with and without aura. Neurology 56:1028-1032

18. Kaunisto MA, Tikka PJ, Kallela M et al (2005) Chromosome 19p13 loci in Finnish migraine with aura families. Am J Med Genet B Neuropsychiatr Genet 132:85-89

19. Kors EE, van den Maagdenberg AM, Plomp JJ et al (2002) Calcium channel mutations and migraine. Curr Opin Neurol 15:311-316

20. Colson NJ, Lea RA, Quinlan S et al (2004) The estrogen receptor 1 G594A polymorphism is associated with migraine susceptibility in two independent case/control groups. Neurogenetics 5:129-133

21. Lea RA, Ovcaric M, Sundholm J et al (2004) The methylenetetrahydrofolate reductase gene variant $\mathrm{C} 677 \mathrm{~T}$ influences susceptibility to migraine with aura. BMC Med 12:2-3

22. McCarthy LC, Hosford DA, Riley JH et al (2001) Single-nucleotide polymorphism alleles in the insulin receptor gene are associated with typical migraine. Genomics 78:135-149

23. Martelletti P, Lulli P, Morellini M et al (1999) Chromosome 6p-encoded HLADR2 determination discriminates migraine without aura from migraine with aura. Hum Immunol 60:69-74

24. Rainero I, Fasano E, Rubino E et al (2005) Association between migraine and HLA-DRB1 gene polymorphisms. J Headache Pain 6:185-187

25. Rainero I, Grimaldi LME, Salani G et al (2004) Association between the tumor necrosis factor- $\alpha-308 \mathrm{G} / \mathrm{A}$ gene polymorphism and migraine. Neurology 62:141-143

26. Trabace S, Brioli G, Lulli P et al (2002) Tumor necrosis factor gene polymorphism in migraine. Headache 42:341-345

27. Lulli P, Trabace S, Morellini M et al (2005) Cytotoxic T lymphocyte antigen 4 polymorphism $49(\mathrm{~A}>\mathrm{G})$ and migraine. J Headache Pain 6:188-190

28. Rubino E, Ferrero M, Rainero I et al (2007) Association of the C677T polymorphism in the MTHFR gene with migraine: a meta-analysis. Cephalalgia (in press)
29. Kaunisto MA, Kallela M, Hämäläinen E et al (2006) Testing of variants of the MTHFR and ESR1 genes in 1798 Finnish individuals fails to confirm the association with migraine with aura. Cephalalgia 26:1462-1472

30. Peroutka SJ, Price SC, Wilhoit TL, Jones KW (1998) Comorbid migraine with aura, anxiety, and depression is associated with dopamine D2 receptor (DRD2) NcoI alleles. Mol Med 4:14-21

31. Paterna S, Di Pasquale P, D'Angelo A et al (2000) Angiotensin-converting enzyme gene deletion polymorphism determines an increase in frequency of migraine attacks in patients suffering from migraine without aura. Eur Neurol 43133-43136

32. De Filippis S, Salvatori E, Farinelli I et al (2007) Chronic daily headache and medication overuse headache: clinical read-outs and rehabilitation procedures. Clin Ter 158:343-347

33. Cevoli S, Mochi M, Scapoli C et al (2006) A genetic association study of dopamine metabolism-related genes and chronic headache with drug abuse. Eur J Neurol 13:1009-1013

34. Di Lorenzo C, Sances G, Di Lorenzo G et al (2007) The wolframin His611Arg polymorphism influences medication overuse headache. Neurosci Lett 13:179-184

35. Collins FS (1999) Genetics: an explosion of knowledge is transforming clinical practice. Geriatrics 54:41-47

36. Weinshilboum R (2003) Inheritance and drug response. N Engl J Med 348:529-537

37. Evans WE, McLeod HL (2003) Pharmacogenomics - drug disposition, drug targets, and side effects. N Engl J Med 6:538-549

38. Severino G, Chillotti C, Stochino ME, Del Zompo M (2003) Pharmacogenomics: state of the research and perspectives in clinical application. Neurol Sci 24:S146-S148

39. Stam AH, Haan J, Frants RR et al (2005) Migraine: new treatment options from molecular biology. Expert Rev Neurother 5:653-661

40. Ferrari MD, Haan J (2002) The genetics of migraine: implication for treatment approaches. J Neural Transm Suppl (63):111-127
41. Fernandez F, Colson NJ, Griffiths L (2007) Pharmacogenetics of migraine: genetic variants and their potential role in migraine therapy. Pharmacogenomics 8:609-622

42. van den Maagdenberg AM, Haan J, Terwindt GM, Ferrari MD (2007) Migraine: gene mutations and functional consequences. Curr Opin Neurol 20:299-305

43. Montagna P, Pierangeli G, Cevoli S et al (2005) Pharmacogenetics of headache treatment. Neurol Sci 26:S143-S147

44. Ophoff RA, van den Maagdenberg AM, Roon KI et al (2001) The impact of pharmacogenetics for migraine. Eur J Pharmacol 413:1-10

45. Montagna $P$ (2007) Recent advances in the pharmacogenomics of pain and headache. Neurol Sci 28[Suppl 2]:S208-S212

46. Colombo B, Annovazzi PO, Comi G (2004) Therapy of primary headaches: the role of antidepressants. Neurol Sci 3:S171-S175

47. Tfelt-Hansen P (2007) Acute pharmacotherapy of migraine, tension-type headache, and cluster headache. J Headache Pain 8:127-134

48. Maassen VanDenBrink A, Vergouwe MN, Ophoff RA et al (1998) 5-HT1B receptor polymorphism and clinical response to sumatriptan. Headache 38:288-291

49. Smeraldi E, Zanardi R, Benedetti $\mathrm{F}$ et al (1998) Polymorphism within the promoter of the serotonin transporter gene and antidepressant efficacy of Fluvoxamine. Mol Psychiatry 3:508-511

50. Cherchi A, Stochino ME, Piccardi MP, Del Zompo M (2001) Role of dopaminergic system in migraine. $\mathrm{J}$ Headache Pain 2:S47

51. Del Zompo M, Cherchi A, Palmas MA et al (1998) Association between dopamine receptor genes and migraine without aura in a Sardinian sample. Neurology 51:781

52. Phillips KA, Veenstra DL, Oren E et al (2001) Potential role of pharmacogenomics in reducing adverse drug reactions. A systematic review. JAMA 286:2270-2279

53. International HapMap Consortium (2005) A haplotype map of the human genome. Nature 437:1299-1320

54. Nicolas P, Sun F, Li LM (2006) A model-based approach to selection of tag SNPs. BMC Bioinform 7:303 